\title{
Patients with primary sclerosing cholangitis require more sedation during endoscopic retrograde cholangiography
}

\section{(๑) $\odot \odot$}

\author{
Authors \\ Carsten Keil ${ }^{1}$, Lukas Aguirre Dávila², Theodor Framke², \\ Henrike Lenzen ${ }^{1}$, Michael P. Manns ${ }^{1}$, Tim O. Lankisch ${ }^{1}$, \\ Torsten Voigtländer ${ }^{1}$ \\ Institutions \\ 1 Department of Gastroenterology, Hepatology and \\ Endocrinology, Hannover Medical School, Hannover, \\ Germany \\ 2 Institute for Biostatistics, Hannover Medical School, \\ Hannover, Germany
}

submitted 1.6.2016

accepted after revision 30.12.2016

\section{Bibliography}

DOI http://dx.doi.org/10.1055/s-0043-104858 |

Endoscopy International Open 2017; 05: E315-E320

(c) Georg Thieme Verlag KG Stuttgart · New York

ISSN 2364-3722

Corresponding author

Torsten Voigtländer, MD, Department of

Gastroenterology, Hepatology and Endocrinology,

Medical School Hannover, Carl-Neuberg Str. 1,

30625 Hannover, Germany

Fax: +49-511-5324896

voigtlaender.torsten@mh-hannover.de

\section{ABSTRACT}

Background and study aims Patients with primary sclerosing cholangitis (PSC) require repeated endoscopic retrograde cholangiography (ERC). Our aim was to evaluate whether patients with PSC require higher doses of sedation during ERC.

Patients and methods We retrospectively analyzed all patients undergoing ERC from 2006 to 2013 who received conscious sedation with propofol and midazolam. The duration of the intervention and a potential progression of propofol consumption or intervention time by visit number were analyzed. Univariable and multivariable analyses were performed to identify independent factors which influence propofol consumption.

Results A total of 2962 ERC procedures were performed in 1211 patients. Patients with PSC $(n=157)$ underwent 461 ERC procedures whereas patients without PSC $(n=$ 1054 ) had 2501 ERC examinations. The total median propofol dose was $450 \mathrm{mg}(290-630 \mathrm{mg})$ for patients with PSC and $300 \mathrm{mg}(200-450 \mathrm{mg})$ for the non-PSC group $(P<0.05)$. The propofol consumption in patients with PSC was increased by a factor of $1.24(P=0.0071)$ independent of intervention time. Younger age ( $<60.8$ years) and duration of the intervention were associated with a higher need for sedation by factors of 1.21 and 1.71, respectively $(P<0.0001)$. The robustness of the results was tested in a sensitivity analysis which confirmed the results $(P<0.0001)$.

Conclusions Patients with PSC may require higher doses of sedation for ERC compared to other patient groups independent of age and duration of ERC. The higher dosage of sedation has to be taken into account when using ERC to treat a patient with PSC.

\section{Introduction}

Primary sclerosing cholangitis (PSC) is a rare chronic liver disease characterized by inflammation and obliterative fibrosis of the intrahepatic and/or extrahepatic bile ducts [1,2]. The progressive fibrosis of the bile ducts may lead to stricture formation, cholestasis and consecutive biliary cirrhosis $[3,4]$. To date, no effective medical treatment is available for patients with PSC [1-4]. Endoscopic procedures form the main part of interventional therapy in order to ensure adequate biliary drainage and to avoid cholestasis-associated liver injury [5-7]. Endoscopic retrograde cholangiography (ERC) is an invasive procedure associated with pain, discomfort, and potentially life-threatening complications $[8,9]$. Consequently, the use of sedation and analgesia is a fundamental aspect of ERC as it may reduce the stress, anxiety, and pain in patients leading to higher acceptability of the procedure $[10,11]$.

Complex endoscopic interventions in patients with PSC may require general anesthesia [12]. However, general anesthesia is not routinely performed in Germany, is expensive and timeconsuming and therefore is only performed in selected patients [12]. Conscious sedation is most often applied in therapeutic ERC and is well tolerated [13]. On the other hand, conscious sedation may progress to general anesthesia in a dose-dependent manner which has to be taken into account [11,13]. As PSC most often presents as progressive disease, repeated endoscopic interventions are mandatory in the majority of patients which underlines the necessity of cost-effective and efficient 
- Table 1 Demographic and baseline characteristics. Patients with and without PSC are compared with regard to age, gender, and duration of first intervention.

\begin{tabular}{|l|c|c|c|}
\hline & PSC & Non-PSC & P value \\
\hline Patients (total observations) & $157(461)$ & $1054(2501)$ \\
\hline Observations per patient, $\mathrm{n}$ (range) & $2(1-4)$ & $1(1-3)$ & - \\
\hline Age, mean \pm SD, years & $41.2 \pm 12$ & $61.1 \pm 14.8$ & $<0.00012$ \\
\hline Age<median (60.8 years), $\mathrm{n}(\%)$ & $151(96.2)$ & $465(44.1)$ \\
\hline Female sex, $\mathrm{n}(\%)$ & $41(26.1)$ & $603(57.2)$ & $<0.0001$ \\
\hline Duration of first intervention, mean \pm SD, min & $50.45 \pm 24.77$ & $53.63 \pm 26.88$ \\
\hline
\end{tabular}

examinations $[6,14]$. In clinical practice, patients with PSC seem to have a higher need for sedation for ERC potentially caused by enzyme-induction/inhibition or tolerance $[15,16]$. To verify this clinical observation, we analyzed patients with and without PSC undergoing ERC with conscious sedation.

\section{Patients and methods}

All patients presenting for ERC to the endoscopic unit of Hannover Medical School between 2006 and 2013 were retrospectively analyzed. Patients who underwent an ERC procedure under general anesthesia or who had a history of liver transplantation were excluded from the study as well as patients receiving opioids during the intervention. In the case of repeated endoscopic examinations, all presentations were included in the analysis. Demographic characteristics, duration (min) and time point of the intervention, underlying diseases, and the application rate of the anesthetics (amount of anesthetics) were extracted from the endoscopy database. Deep sedation was performed by intermittent bolus application of propofol with or without midazolam as premedication. No additional application of analgesics was performed. The sedation was administered and monitored by the endoscopists (routinely one endoscopist for examination and one endoscopist for sedation). During ERC, a deep sedation level with maintained cardiovascular and respiratory function was targeted and controlled by gastroenterologists.

The diagnosis of PSC was based on laboratory or clinical findings and typical cholangiographic features in all patients (strictures or irregularity of intrahepatic and/or extrahepatic bile ducts) after exclusion of secondary causes of sclerosing cholangitis. The non-PSC patient subgroup consisted of all patients undergoing ERC fulfilling the inclusion criteria (no PSC, no history of liver transplantation, $>18$ years). All physicians performing ERC at our institution are experienced endoscopists ( $>3$ years of regular ERC performance and $>500$ examinations).

The study was approved by the local institutional Ethics Committee (Ethics Committee of Hannover Medical School).

\section{Statistical analysis}

Baseline characteristics at the time of the first intervention are presented as absolute and relative frequencies for categorical variables and mean \pm standard deviation, unless denoted other- wise. Of main interest was length of the intervention and propofol dose.

The main research hypothesis was that PSC patients require a higher propofol dose than non-PSC patients. We set up a linear mixed model with repeated measurements, where the effect of intervention number was modeled as random and all other effects were modeled as fixed. Propofol dose served as the dependent variable. A logarithmic transformation was applied due to a skewed distribution on the original scale. In a first step, we checked the influence of each independent variable separately in univariable models. Subsequently, all significant $(P<0.05)$ variables were entered into a multivariable model and a backward selection based on the Wald statistic was applied. This approach was chosen in order to find a parsimonious model, including only variables showing an association with the outcome variable in a univariable model as well as after adjusting for other factors. After checking the correlation between log propofol change from baseline and days since first intervention, a progression seemed unlikely. Therefore, we chose a compound symmetry covariance matrix in modeling repeated measurements.

All analyses are of explorative character and hence, no multiplicity correction was applied. SAS 9.3 (SAS Institute Inc., Cary, NC, United States) was used throughout for all statistical analyses.

\section{Results}

A total of 2962 ERC procedures were performed in 1211 patients during the study period ( Table 1 ). Patients with PSC $(n=157)$ underwent 461 ERC procedures whereas patients without PSC $(n=1054)$ had 2501 ERC examinations ( Table 1). PSC patients had a median of two ERC per patient (interquartile range (IQR) 1-4) compared to a median of one ERC examination per patient in the non-PSC group (IQR 1-3). In total, up to 32 repeated measurements are available per patient. The mean age in the PSC cohort was 41.2 years ( \pm 11.95 years) and 61.1 years $( \pm 14.75$ years $)$ in the non-PSC subgroup $(P<0.0001)$. The median body mass index (BMI) was 24.2 (IQR 21.6-26.5) for patients with PSC and 24.7 (IQR 21.5-27.8) for non-PSC patients $(P<0.05)$. The duration of the first endoscopic intervention was $50.45 \mathrm{~min}( \pm 24.77 \mathrm{~min})$ for patients with PSC and $53.63 \mathrm{~min}( \pm 26.88 \mathrm{~min})$ for non-PSC patients $(P=0.1619)$. Addi- 


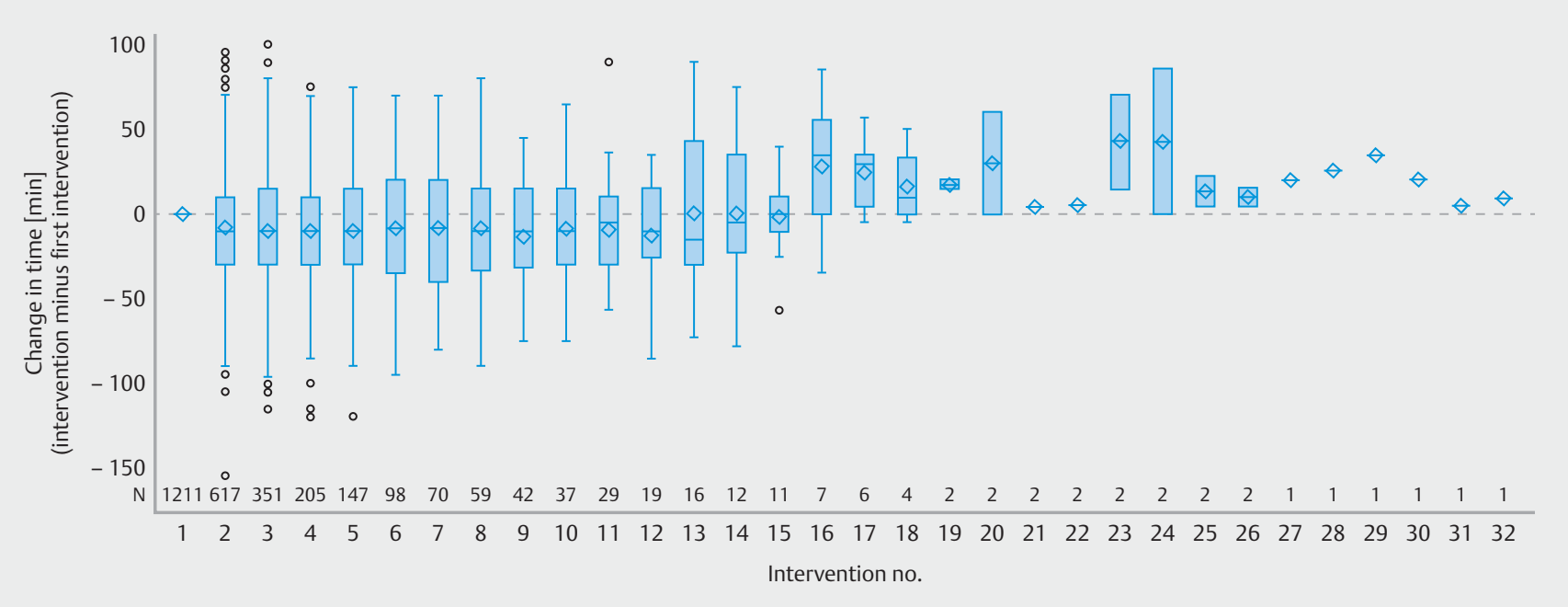

- Fig. 1 Duration of ERC by visit number. No significant change in intervention time by intervention number was apparent. From intervention number 16 , a longer intervention time was detected, but only seven patients had 16 or more interventions.

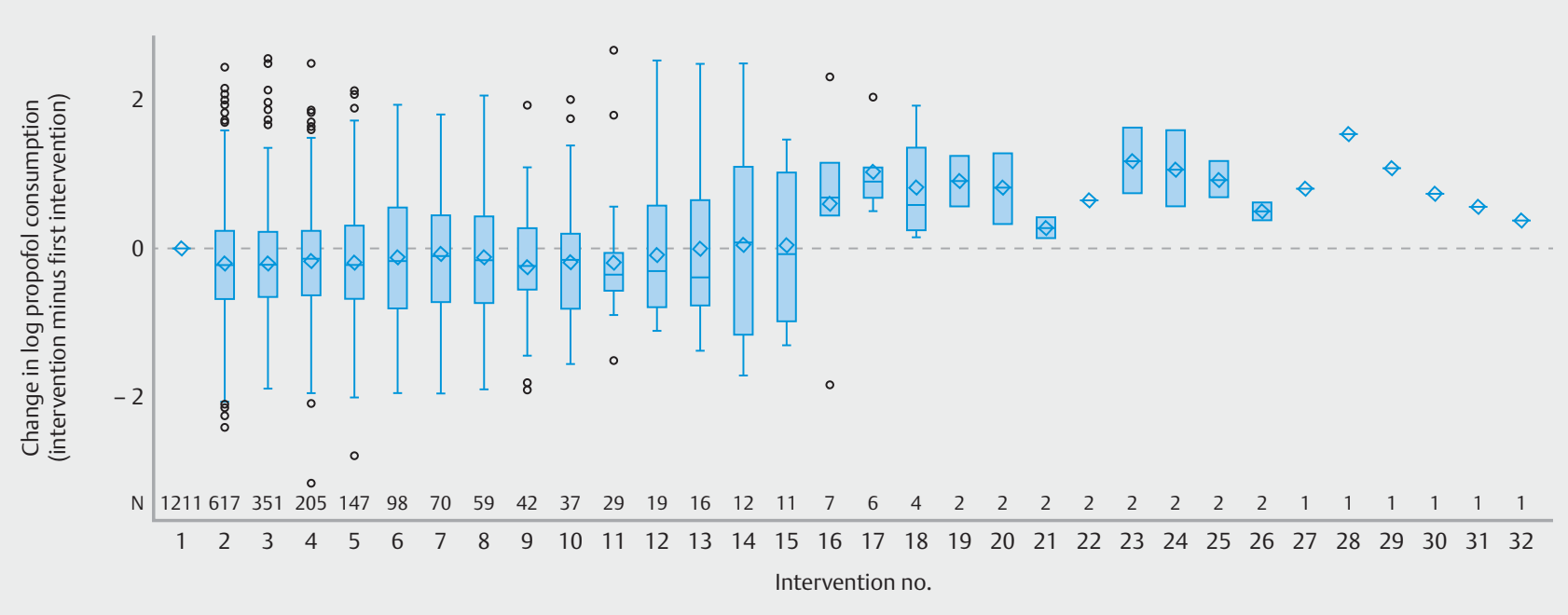

Fig. 2 Propofol dose by visit number. No progression of propofol dose by intervention number was detected (up to 15 interventions).

tional demographic and baseline data are listed in $>$ Table 1. The indications for ERC in the non-PSC subgroup were malignancies $(44.4 \%)$, gall or pancreatic stones $(21.8 \%)$, pancreatitis (11.3\%), benign bile duct stenoses (7.9\%), and others (14.6\%).

Patients with and without PSC received a median of $5 \mathrm{mg}$ midazolam per ERC (IQR 5-5 mg for PSC and 3.15-5 mg for non-PSC patients, respectively; $P=0.0001)$. The analysis of the duration of ERC procedures showed no progression over time by visit number for all patients (up to 15 interventions) ( $\triangleright$ Fig. 1). The total median propofol dose was $450 \mathrm{mg}(290-$ $630 \mathrm{mg}$ ) for patients with PSC and $300 \mathrm{mg}(200-450 \mathrm{mg})$ for the non-PSC group $(P<0.05)$. The propofol consumption was equal over time and revealed no progression in the case of repeated ERC procedures for all patients (up to 15 interventions) ( $\triangleright$ Fig.2). The median time interval between repeat ERC examinations was 62 days (IQR 20-97 days). To exclude a potential progression of the propofol consumption or duration of ERC as a function of the time interval between repeat examinations, a pairwise linear correlation analysis was performed. No relevant correlation was identified for each of the first 11 visits $(r<0.5)$

\section{( Table 2).}

In a univariable analysis, presence of PSC $(P=0.0048)$, age $(P<0.0001)$, gender $(P=0.0234)$, and duration of the endoscopic intervention $(P<0.0001)$ showed a significant influence on propofol consumption ( $\downarrow$ Table 3 ). Multivariable analysis verified the presence of PSC (Exponential function (Exp) (Estimate) $1.2358 ; 95 \% \mathrm{Cl}: 1.1158 ; 1.3686(P=0.0071))$, age (Exp (Estimate) $0.7889 ; 95 \% \mathrm{Cl}: 0.7528 ; 0.8267(P<0.0001))$, and log duration of the intervention (Exp (Estimate) 1.7098; $95 \%$ Cl: 1.6769; $1.7433(P<0.0001))$ as independent variables which influence propofol consumption ( $\triangleright$ Table 3 ). Exp (Estimate) denotes the back-transformation on a multiplicative scale, e.g. the existence of PSC leads on average (geometric mean) to an approximately $24 \%$ higher propofol consumption 
- Table 2 Pairwise linear correlation analysis. To exclude a potential progression of propofol consumption or duration of ERC as a function of the time interval between repeat examinations, a pairwise linear correlation analysis was performed. No relevant correlation was identified $(r<0.5)$.

\begin{tabular}{|l|l|l|}
\hline & Days since first intervention & days since prior intervention \\
\hline Logarithmic propofol change & $r=0.18138 ; P<0.0001$ & $r=0.08910 ; P=0.0002$ \\
\hline Duration change & $r=0.10687 ; P<0.0001$ & $r=0.03452 ; P=0.1488$ \\
\hline
\end{tabular}

- Table 3 Univariable and multivariable analysis to identify factors which impact on propofol dosage. Diagnosis of PSC, age, and duration of ERC were independently associated with propofol consumption.

\begin{tabular}{|l|l|l|l|l|}
\hline & \multicolumn{2}{|l|}{ Univariate } & \multicolumn{2}{l|}{ Multivariate (backward selected) } \\
\hline Variable reference & Estimate 95\%Cl & P value & Estimate 95\%Cl & Exp (Estimate) \\
\hline PSC vs no PSC & $0.3163(0.1834 ; 0.4491)$ & 0.0048 & $0.2117(0.1096 ; 0.3138)$ & $1.2358(1.1158 ; 1.3686)$ \\
\hline $\begin{array}{l}\text { Age }>60.80 \text { years old vs } \\
\text { young }\end{array}$ & $\begin{array}{l}-0.2617(-0.3224 ; \\
-0.2010)\end{array}$ & $<0.0001$ & $\begin{array}{l}-0.2371(-0.2839 ; \\
-0.1903)\end{array}$ & $0.7889(0.7528 ; 0.8267)$ \\
\hline Sex, female vs male & $\begin{array}{l}-0.06968(-0.1299 ; \\
-0.00945)\end{array}$ & 0.0234 & & $<0.0001$ \\
\hline $\begin{array}{l}\text { Log duration of interven- } \\
\text { tion }\end{array}$ & $0.7680(0.7394 ; 0.7966)$ & $<0.0001$ & $0.7738(0.7458 ; 0.8018)$ & $1.7098(1.6769 ; 1.7433)$ \\
\hline
\end{tabular}

- Table 4 Sensitivity analysis restricted to the first presentation for ERC. The robustness of the results from the multivariable analysis was verified.

\begin{tabular}{|l|c|c|}
\hline Variable reference & Estimate $95 \% \mathrm{Cl}$ & $P$ value \\
\hline PSC vs no PSC & $0.1711(0.1096 ; 0.3138)$ & $<0.0001$ \\
\hline Age $>60.80$ years old vs young & $-0.2604(-0.2839 ;-0.1903)$ & $<0.0001$ \\
\hline Log duration of intervention & $0.7745(0.7458 ; 0.8018)$ & $<0.0001$ \\
\hline
\end{tabular}

than in non-PSC patients, provided that all other variables are held constant. The interpretation of the log-transformed independent variable duration of intervention differs slightly: Doubling of the duration of the intervention leads to an increase of $70.98 \%$ in propofol consumption, given that all other variables in the model are kept constant. In order to test the robustness of the multivariable analysis, a sensitivity analysis restricted to the first presentation of all patients was performed. The aforementioned variables (PSC, age, and duration of the intervention) were confirmed as independent factors which influence propofol consumption $(P<0.0001)$ ( $\triangleright$ Table 4$)$. After checking the residuals of the final model, a normality assumption seems plausible.

\section{Discussion}

Sedation for ERC has positive effects of reducing anxiety and pain in patients and is useful for the completion rate, quality of endoscopic procedures, and treatment outcomes for endoscopists, especially in the setting of repeated and complex interventions $[10,11]$. Nevertheless, sedation is associated with potential complications, particularly cardiovascular events [13, 17]. At our institution, application of sedative agents for ERC is routinely performed by a gastroenterologist.

In our analysis, we show that patients with PSC need 24\% more sedative agents (propofol) to achieve favorable endo- scopic conditions and to perform an ERC compared to non-PSC patients. Multivariable analysis verified that the presence of PSC remains an independent predictor of propofol dose even after adjusting for age and duration of the endoscopic intervention. In addition, patients with PSC received significantly more midazolam per ERC which endorses the finding of an increased need for sedation to perform an ERC. From a clinical point of view, one may speculate that the increased need for sedation to perform an ERC in patients with PSC is caused by the younger age and the predominant male sex of the patients compared to the non-PSC group. However, these considerations are very unlikely as the multivariable analysis shows that propofol dose was independent of intervention time and age/ sex of the patients.

The mechanisms leading to the higher demand of sedation in patients with PSC remain speculative and cannot be answered by this observational study. One may speculate that altered enzymatic pathways in the liver may contribute to this phenomenon as propofol is predominantly metabolized in the liver and is excreted by the kidneys [10]. Moreover, the composition of bile differs in chronic biliary diseases which may lead to alterations in bile acid synthesis, conjugation, transport, and metabolism $[18,19]$. These factors may be of importance in patients with PSC. Further studies are required to address this question.

The major factors affecting the pharmacokinetic profile and clinical effects of propofol are gender, weight, and age [10]. 
Age-related changes in the need for sedation for different endoscopic procedures are well described $[13,20,21]$. A higher susceptibility for anesthetic drugs in elderly patients is caused by a physiological decline in hepatic volume and function, as well as decreased hepatic blood flow leading to a slower metabolism of many intravenous drugs used for anesthetic purposes [20]. Furthermore, reduced albumin levels may result in an increase in the free concentration of protein bound drugs such as propofol. All lipophilic drugs may have a prolonged effect due to the greater volume of distribution into larger fat reserves in the elderly patient. Thus, the effective concentration of anesthetic agents is increased due to distribution into a smaller initial volume, followed by a slower redistribution and clearance $[20,22,23]$.

In our patient cohort, older patients (as dichotomized by the median of 60.8 years) needed $21 \%$ less propofol for the completion of ERC compared to the younger patients. Similar findings have been published in earlier studies emphasizing that, due to the possible increase in adverse events in elderly patients, a reduction in the maintenance dose of propofol should be performed $[23,24]$. The volume of distribution is increased in patients with a higher body mass index (BMI) for lipid soluble agents such as propofol resulting in the application of higher doses of the agent to achieve favorable endoscopic conditions [25]. In our cohort, patients with PSC showed a lower BMI compared to non-PSC patients. Nevertheless, patients with PSC were in need of higher doses of propofol emphasizing that the calculated difference in propofol dosage might even be underestimated in patients with PSC.

As expected, duration of ERC had a significant influence on the propofol dose. A doubling of the intervention time led to an increase in the propofol dose by a factor of 1.71 . For experienced endoscopists, intervention time is a surrogate parameter of the complexity of an endoscopic intervention. In both groups, the intervention time to complete an ERC was the same, which emphasizes that an increased need for sedation in patients with PSC due to the complexity of the intervention is very unlikely.

In our study, similar doses of propofol were applied for the completion of ERC compared to previous studies which included continuous and intermittent application of propofol [2628]. Over recent years, propofol has been increasingly used as sedation for ERC showing a low incidence of serious adverse events and a significantly shorter recovery time compared to conventional sedation [10,26-28]. Our study was not designed to analyze sedation-associated adverse events in patients undergoing ERC and did not analyze the post-interventional recovery time as this was not routinely documented. Moreover, the retrospective study design did not allow to exclude potential confounders such as concomitant use of nonsteroidal anti-inflammatory drugs, selective serotonin reuptake inhibitors, or alcohol consumption of the patients which may have had an impact on the required dose of sedation for ERC. In addition, the type of bile duct intervention was not included in our analysis. The type of procedure might influence the need for propofol dosage during ERC. As our study was retrospective, we unfortunately could not present reliable data to answer this question. As an example, the performance of dilatation therapy is not standardized which means that different investigators perform the intervention in a different way (dilatation time, number of dilatations, different balloon catheters). Moreover, this information was not routinely documented in our endoscopic data base which hindered the analysis. In contrast, the dose of anesthetic drugs is a numeric variable which does not require subjective interpretation and could be adequately retrieved from our data base. Our study is a first preliminary step to analyze whether patients with PSC need more sedation for the performance of ERC. These questions have to be answered in a prospective setting and are under ongoing investigation.

In summary, our study shows that patients with PSC may require more sedation during ERC independent of age and duration of ERC compared to other patients. The higher dosage of sedation has to be taken into account when using ERC to treat a patient with PSC.

\section{Competing interests}

None

\section{References}

[1] Trauner M, Halilbasic E, Baghdasaryan A et al. Primary sclerosing cholangitis: new approaches to diagnosis, surveillance and treatment. Dig Dis 2012; 30: (Suppl. 01): 39-47

[2] Karlsen TH, Boberg KM. Update on primary sclerosing cholangitis. J Hepatol 2013; 59: 571 - 582

[3] Boberg KM, Lind GE. Primary sclerosing cholangitis and malignancy. Best Pract Res Clin Gastroenterol 2011; 25: 753-764

[4] Karlsen TH, Vesterhus M, Boberg KM. Review article: controversies in the management of primary biliary cirrhosis and primary sclerosing cholangitis. Aliment Pharmacol Ther 2014; 39: 282 - 301

[5] Voigtländer T, Lankisch TO. Endoscopic diagnosis of cholangiocarcinoma: From endoscopic retrograde cholangiography to bile proteomics. Best Pract Res Clin Gastroenterol 2015; 29: 267 - 275

[6] Chapman MH, Webster G], Bannoo S et al. Cholangiocarcinoma and dominant strictures in patients with primary sclerosing cholangitis: a 25-year single-centre experience. Eur J Gastroenterol Hepatol 2012; 24: $1051-1058$

[7] Modha K, Navaneethan U. Diagnosis and management of primary sclerosing cholangitis - perspectives from a therapeutic endoscopist. World J Hepatol 2015; 7: 799-805

[8] Moon JH, Choi HJ, Lee YN. Endoscopic retrograde cholangiopancreatography. Gastrointest Endosc 2014; 80: 388-391

[9] Chavalitdhamrong D, Donepudi S, Pu L et al. Uncommon and rarely reported adverse events of endoscopic retrograde cholangiopancreatography. Dig Endosc 2014; 26: 15-22

[10] Ferreira AO, Cravo M. Sedation in gastrointestinal endoscopy: Where are we at in 2014? World J Gastrointest Endosc 2015; 7: $102-109$

[11] Obara K, Haruma K, Irisawa A et al. Guidelines for sedation in gastroenterological endoscopy. Dig Endosc 2015; 27: 435-449

[12] Raymondos K, Panning B, Bachem I et al. Evaluation of endoscopic retrograde cholangiopancreatography under conscious sedation and general anesthesia. Endoscopy 2002; 34: 721 - 726 
[13] Cheriyan DG, Byrne MF. Propofol use in endoscopic retrograde cholangiopancreatography and endoscopic ultrasound. World J Gastroenterol 2014; 20: $5171-5176$

[14] Thosani N, Banerjee S. Endoscopic retrograde cholangiopancreatography for primary sclerosing cholangitis. Clin Liver Dis 2014; 18 : 899-911

[15] Sweeney BP, Bromilow J. Liver enzyme induction and inhibition: implications for anaesthesia. Anaesthesia 2006; 61: 159-177

[16] Tanaka E. Clinically important pharmacokinetic drug-drug interactions: role of cytochrome P450 enzymes. J Clin Pharm Ther 1998; 23 : $403-416$

[17] Andriulli A, Loperfido S, Napolitano G et al. Incidence rates of postERCP complications: a systematic survey of prospective studies. Am J Gastroenterol 2007; 102: $1781-1788$

[18] Li T, Apte U. Bile acid metabolism and signaling in cholestasis, inflammation, and cancer. Adv Pharmacol 2015; 74: 263 -302

[19] Trauner M, Halilbasic E, Kazemi-Shirazi L et al. Therapeutic role of bile acids and nuclear receptor agonists in fibrosing cholangiopathies. Dig Dis 2014; 32: 631-636

[20] Rivera R, Antognini JF. Perioperative drug therapy in elderly patients. Anesthesiology 2009; 110: 1176-1181
[21] Travis AC, Pievsky D, Saltzman JR. Endoscopy in the elderly. Am J Gastroenterol 2012; 107: 1495-1501

[22] Rooke GA. Cardiovascular aging and anesthetic implications. J Cardiothorac Vasc Anesth 2003; 17: 512-523

[23] Shafer SL. The pharmacology of anesthetic drugs in elderly patients. Anesthesiol Clin North America 2000; 18: 1-29, v

[24] Schnider TW, Minto CF, Shafer SL et al. The influence of age on propofol pharmacodynamics. Anesthesiology 1999; 90: 1502-1516

[25] Vargo JJ. Procedural sedation and obesity: waters left uncharted. Gastrointest Endosc 2009; 70: 980 - 984

[26] Kongkam P, Rerknimitr R, Punyathavorn S et al. Propofol infusion versus intermittent meperidine and midazolam injection for conscious sedation in ERCP. J Gastrointest Liver Dis 2008; 17: 291 - 297

[27] Jung M, Hofmann C, Kiesslich R et al. Improved sedation in diagnostic and therapeutic ERCP: propofol is an alternative to midazolam. Endoscopy 2000; 32: 233-238

[28] Vargo J], Zuccaro GJr, Dumot JA et al. Gastroenterologist-administered propofol versus meperidine and midazolam for advanced upper endoscopy: a prospective, randomized trial. Gastroenterology 2002; 123: $8-16$ 